\title{
Estimating risks of importation and local transmission of Zika virus infection
}

Kyeongah Nah, Kenji Mizumoto, Yuichiro Miyamatsu, Yohei Yasuda, Ryo Kinoshita, Hiroshi Nishiura

Background. An international spread of Zika virus (ZIKV) infection has attracted global attention. ZIKV is conveyed by mosquito vector, Aedes species which also act as the vector species of dengue and chikungunya viruses. Methods. Arrival time of ZIKV importation (i.e. the time at which the first imported case was diagnosed) in each imported country was collected from publicly available data source. Employing a survival analysis model in which the hazard is an inverse function of the effective distance as informed by the airline transportation network data, and using dengue and Chikungunya virus transmission data, risks of importation and local transmission were estimated. Results. A total of 78 countries with imported case(s) have been identified, with the arrival time ranging from 1 to 44 weeks since the first ZIKV was identified in Brazil, 2015. Whereas the risk of importation was well explained by the airline transportation network data, the risk of local transmission appeared to be best captured by additionally accounting for the presence of dengue and Chikungunya viruses. Discussion. The risk of importation may be high given continued global travel of mildly infected travelers, but, considering that the public health concerns over ZIKV infection stems from microcephaly, it is more important to focus on the risk of local and widespread transmission that could involve pregnant women. The predicted risk of local transmission was frequently seen in tropical and subtropical countries with dengue or Chikungunya epidemic experience. 


\section{Estimating risks of importation and local transmission of}

\section{Zika virus infection}

3

4 Kyeongah Nah ${ }^{1,2,4}$, Kenji Mizumoto ${ }^{1,3,4}$, Yuichiro Miyamatsu ${ }^{1,2,4}$, Yohei Yasuda ${ }^{1}$, Ryo

5 Kinoshita $^{1,2,4}$, Hiroshi Nishiura ${ }^{1,2,4}$

$6{ }^{1}$ Graduate School of Medicine, The University of Tokyo, Tokyo, Japan

$7 \quad 2$ CREST, Japan Science and Technology Agency, Saitama, Japan

$8{ }^{3}$ Graduate School of Arts and Sciences, The University of Tokyo, Tokyo, Japan

$9{ }^{4}$ Graduate School of Medicine, Hokkaido University, Sapporo, Japan

11 Corresponding Author:

12 Hiroshi Nishiura

13 Kita 15 Jo Nishi 7 Chome, Kita-ku, Sapporo-shi, Hokkaido 060-8638, Japan

14 Email address: nishiurah@gmail.com 


\section{Abstract}

17 Background. An international spread of Zika virus (ZIKV) infection has attracted global

18 attention. ZIKV is conveyed by mosquito vector, Aedes species which also act as the vector

19 species of dengue and chikungunya viruses.

20 Methods. Arrival time of ZIKV importation (i.e. the time at which the first imported case was

21 diagnosed) in each imported country was collected from publicly available data source.

22 Employing a survival analysis model in which the hazard is an inverse function of the effective

23 distance as informed by the airline transportation network data, and using dengue and

24 Chikungunya virus transmission data, risks of importation and local transmission were estimated.

Results. A total of 78 countries with imported case(s) have been identified, with the arrival time ranging from 1 to 44 weeks since the first ZIKV was identified in Brazil, 2015. Whereas the risk of importation was well explained by the airline transportation network data, the risk of local transmission appeared to be best captured by additionally accounting for the presence of dengue and Chikungunya viruses.

Discussion. The risk of importation may be high given continued global travel of mildly infected travelers, but, considering that the public health concerns over ZIKV infection stems from microcephaly, it is more important to focus on the risk of local and widespread transmission that could involve pregnant women. The predicted risk of local transmission was frequently seen in tropical and subtropical countries with dengue or Chikungunya epidemic experience. 


\section{Introduction}

37 Zika virus belongs to the virus family Flaviviridae and genus Flavivirus, initially isolated from

38 Zika forest of Uganda (Dick, 1952). Related to other arboviruses such as chikungunya, dengue,

39 yellow fever, Japanese encephalitis and West Nile viruses, the virus had circulated in sub-

40 Saharan African, South and Southeast Asian and South Pacific countries. Once reaching to South

41 America, an international spread of Zika virus (ZIKV) infection has attracted global attention, as

42 a large epidemic of ZIKV infection in Brazil was considered to have been associated with

43 remarkable increase in the number of microcephaly cases that are suspected to have been caused

44 by ZIKV infection among pregnant women (Staples et al., 2016). The causal relationship

45 between ZIKV infection and microcephaly has yet to be fully established, but increasing

46 scientific evidence strongly supports the epidemiological link of ZIKV with microcephaly

47 (Mlakar et al., 2016; Brasil et al., 2016; Nishiura et al., 2016b). Considering the expected impact

48 on neurological manifestations including microcephaly and Guillain-Barré Syndrome (GBS), the

49 World Health Organization issued the Public Health Emergency of International Concern

50 (PHEIC) on 1 February 2016.

51

ZIKV shares self-limiting clinical signs and symptoms with dengue virus (DENV) and

52 Chikungunya virus (CHIKV) (Ioos et al., 2014). Moreover, these viruses are also conveyed by

53 the common mosquito vector, Aedes species. Technically, the infected cases could be observed

54 in any part of the world in the presence of Aedes species, but the risk of transmission in Latin

55 American and Caribbean countries has been anticipated to be high (Musso, Cao-Lormeau \&

56 Gubler, 2015; Bogoch et al., 2016). The transmission potential of ZIKV infection has been

57 shown to be comparable to those of DENV and CHIKV (Nishiura et al., 2016a). Considering that 
58 there are no specific treatments for these arboviruses, it is of utmost importance to intervene

59

60

61

62

63

64

transmission by preventing mosquito biting (e.g. the use of repellent) or controlling Aedes species, most notably, targeting highly invasive species including Aedes aegypti and Aedes albopictus. The control option of Aedes spp. includes source reduction (i.e. removal of water and covering water storage containers), the use of ovitrap, chemical control, bioinsecticides and biological control (e.g. mosquitofish)(Anonymous, 2016), while recent approaches include transgenic and symbiont-based approaches and the use of plant-borne molecules (Adelman \& Tu, 2016; Benelli, 2016; Benelli \& Mehlhorn, 2016).

To plan for forthcoming potential local transmission at each country level, it is fruitful to understand the actual risk of Zika virus epidemic in a quantitative manner. The present real-time study aimed to identify countries at high risk of importing ZIKV infection and also of local transmission, as a part of risk assessment practice and for improved understanding of the predicted risk of the spread, with distinct differentiation of ZIKV importation alone from that of local transmission.

\section{Materials \& Methods}

\section{Epidemiological data}

Arrival times of ZIKV importation, i.e., the time at which first ZIKV case was imported, in each imported country was collected from publicly available data sources (see Supplementary Material). The criterion to include a country as imported was the presence of a report based on confirmatory diagnosis made either by serological testing or detection of virus RNA. When ZIKV was isolated from mosquitoes or primates other than humans, it was considered that the country had already imported the virus, because the virus isolation implies that the local 
80

81

82

transmission had taken place. For each imported country, the arrival time was defined as the week in which the importation event had happened. When monthly or yearly data were only available or when a seroepidemiological study identified the local transmission, the median week was used as the week of importation. The latest time at which the importation week was systematically examined was 31 January 2016.

In addition to the importation data, we collected the list of countries with local transmission of ZIKV from European Centres for Disease Control (ECDC, 2016) and Gatherer and Kohl (2016). ECDC resource has specified countries or territories with reported confirmed autochthonous cases of ZIKV infection in the past 9 months. Since ECDC (2016) list misses countries that experienced local transmission in 2014 or earlier, we extracted the list of those earlier countries from Gatherer and Kohl (2016). Again, the virus isolation from mosquitoes or primates other than humans was regarded as a signature of local transmission.

As additional input of prediction models, an Open Source airline transportation network data were extracted. Using Global Flights Network (2016) derived from OpenFlights database as on 10 November 2014 (Contentshare, 2016), we obtained the total number of flight routes between each pair of countries (total 230 airports and 4,600 flight routes). Furthermore, countryspecific presence of Aedes species, especially focusing on Aedes albopictus, Aedes aegypti and Aedes africana, was explored (Kraemer et al., 2015; The Walter Reed Biosystematics Unit, 2016). The list of countries with at least one of abovementioned Aedes species was prepared, because the local human-vector-human transmission of ZIKV infection occurs only where the vector species are present. Similarly, we also retrieved the country-specific data regarding DENV and CHIKV epidemic (CDC 2016a; 2016b), because they share major vector species with ZIKV and their epidemics indicate that the vector species have been actively involved in local 
103 transmission. Moreover, it has been explicitly discussed that international spread of Zika virus

104 followed the geographic path of CHIKV (Musso, Cao-Lormeau \& Gubler, 2015). The presence 105 of Aedes species, DENV and CHIKV were all dealt with as dichotomous variable.

106 Prediction model

We estimated the risk of importing ZIKV and that of local transmission using a survival analysis model. Let $T$ be a continuous random variable with probability density function of the time from importation in Brazil to importation in country $j, f_{j}(t)$, and cumulative distribution function $F_{j}(t)=\operatorname{Pr}(T<t)$. The function $F_{\mathrm{j}}(t)$ describes the probability that ZIKV has been already imported to a country $j$ by time $t$. The time $t=0$ corresponds to the time at which ZIKV infection was first recognized in Brazil and started to rapidly spread across countries (i.e. week 12 of 2015). We parameterize the hazard function $\lambda(t)$ using the effective distance, the metric derived from the airline transportation network (Contentshare, 2016). Let $\left\{n_{1}, n_{2}, \ldots n_{l}\right\}$ be the sequence of transit countries at which a traveler starting from country $n_{1}$ (with a destination $n_{l}$ ) stops over. The length of path of that travel is $l$. The effective length of the path, $d \mathrm{n}_{1} \mathrm{n}_{1}$, is defined 117 by

$$
d_{n 1 n l}=l-\log \prod_{k=1}^{l-1} P_{n_{k+1} n_{k}^{\prime}}
$$
where $P_{j i}$ denotes the conditional probability that an individual that left $i$ moves to $j$. Assuming that the number of passengers is identical among all international flights, the transition matrix is

121 calculated as $P_{j i}=\frac{w_{j i}}{\sum_{k} w_{k i}}$, where $w_{k i}$ is the number of direct flights from country $i$ to country $k$ per 122 unit time derived from airline transportation network data (Contentshare, 2016). Finally, the 123 effective distance $m_{j}$ of a country $j$ from the country of origin (i.e. Brazil) is calculated as the 
124 minimum of the all possible effective lengths of path that goes from the origin country to the

125 country $j$. The effective distance has been known as an excellent predictor of the arrival time of

126 SARS (severe acute respiratory syndrome) and influenza pH1N1 2009 (Brockmann \& Helbing

127 2013). Considering that the effective distance is a critical indicator of the risk of importation, we

128 assume that the hazard function is an inverse of the effective distance, i.e.,

$$
\lambda_{j}(t)=\frac{k}{m_{j}}
$$

130

131

132

$$
f_{j}(t)=\lambda_{j}(t) \exp \left(-\int_{0}^{t} \lambda_{j}(s) d s\right)
$$

Modeling $f_{j}(t)$ in this way, the mean arrival time of the ZIKV to a country $j$ would be proportional to the effective distance from the origin of spread (i.e. Brazil in our case study).

Using the calculated risk of importation, we subsequently model the country specific risk of local transmission. Let $p_{j}$ be the conditional probability of ZIKV transmission given an importation event in a country $j$. Since our time scale of examining the risk of importation is much longer than the generation time of ZIKV infection, we ignore the time-lag that is required for observing the transmission cycle, and the risk of the local transmission of ZIKV in a country $j$ is modeled as

$$
g_{j}(\mathrm{t})=p_{j} f_{j}(t)
$$


142 In order to estimate $p_{j}$, the conditional probability of experiencing local transmission, we use

143 datasets of the presence of Aedes species, CHIKV and DENV in each country. More specifically,

144 we model $p_{j}$ by employing a logit model

$$
p_{j}=\frac{1}{1+\exp \left[-\left(a_{0}+\sum_{i} \beta_{i} x_{j i}\right)\right]}
$$

where $a_{0}$ is an intercept, $x_{j i}$ is the dichotomous variable that describes the presence of Aedes

147 mosquitoes (for $i=1$ ), CHIKV (for $i=2$ ) and DENV (for $i=3$ ) in country $j$, and $\beta_{\mathrm{i}}$ represents the 148 coefficient for corresponding variable $i$.

\section{Parameter estimation and risk assessment}

We estimate parameters $k, a_{0}$ and three $\beta_{\mathrm{i}}$ using a maximum likelihood method. Since we

151

152

153

154

155

156

157

158

159

160

161

162

start the clock from week 12, 2015 originated from Brazil (Campos, Bandeira \& Sardi, 2015), countries that had imported ZIKV in advance (e.g. Africa and South Pacific) were removed before the implementation. In total, we predict the risks of importation and local transmission among a total of 189 countries. The model was fitted at week 46 since importation in Brazil (and the week 46 corresponds to the latest time at which our systematic survey of importation dates was completed in January 2016). Although five countries (Aruba, Trinidad and Tobago, Marshall Islands, Saint Vincent and the Grenadines, and American Samoa) were specified as countries with local transmission by ECDC data source (ECDC, 2016), these countries have experienced importation and confirmation in February 2016, and were thus removed from the list of countries with importation as well as local transmission.

The likelihood of importation risk adhered to survival model. That is, among countries that have already imported ZIKV by then, the arrival time $t_{j}$ was used for parameter estimation. 
163 Countries that have not imported ZIKV by week 46 were dealt with as the censored observation.

164 In other words, the survival time for those countries is considered to be at least as long as the 165 duration of our study. The likelihood function reads

$$
L\left(k, a_{0}, \boldsymbol{\beta}_{\boldsymbol{i}} ; \boldsymbol{t}_{\boldsymbol{j}}, \boldsymbol{x}_{\boldsymbol{j}}, t_{m}\right)=\prod_{j \in A} p_{j} f_{j}\left(t_{j}\right) \prod_{j \in B}\left(1-p_{j}\right) f_{j}\left(t_{j}\right) \prod_{j \in C}\left(1-F_{j}\left(t_{m}\right)\right),
$$

where $A$ represents the set of countries which experienced local transmission of ZIKV by time of observation $t_{m}$ (31 January 2016), $B$ represents countries which imported ZIKV before $t_{m}$ but without local transmission, and $C$ is the set of countries which has not imported by $t_{m}$. Once the parameter $k$ is estimated, we are able to predict the probability of country $j$ to experience local transmission of ZIKV by time $t$ as

$$
G_{j}(t)=p_{j}\left(1-e^{-\frac{k t}{m_{j}}}\right)
$$

We assessed the diagnostic performance of our models in predicting risks of importation and local transmission by employing the Receiver Operating Characteristic (ROC) curve and measuring the Area Under the Curve (AUC) (Greiner, Pfeiffer \& Smith, 2000). The predictors of local transmission in equation (5) were varied by using 1-3 variables (by examining all possible combinations of Aedes, DENV and CHIKV), and we compared AUC to identify the best predictive model. To avoid serious collinearity among Aedes, DENV and CHIKV, Cohen's kappa, an agreement statistic was computed to examine correlations between two dichotomous explanatory variables. For each model, the optimal cut-off value of estimated risk was calculated using Youden index. While model fit was assessed in week 46, the prediction has been made in week 92 that corresponds to the end of 2016. 
183 Results

184 Table 1 shows the list of countries that have experienced ZIKV infection along with arrival time.

185 A total of 78 countries with imported case(s) have been identified, with the arrival time ranging

186 from 52 to 3566 weeks since the first ZIKV was identified in Uganda, 1947. After the

187 introduction to Brazil in early 2015, 39 countries have experienced importation by the end of

188 January 2016. Originally, we had defined $t=0$ as the week of illness onset of first identified ZIKV

189 case in 1947, Uganda (Dick, 1952), but the country was not specified as the origin, because a

190 long time has passed since the emergence and the path of global spread must not have followed a

191 static network. Note that 39 countries which had already imported ZIKV prior to importation

192 event in Brazil were excluded from the analysis.

Using Brazil, 2015 as the origin (Figures 1A and 1C), the model appeared to crudely

capture the risk of importation (AUC $=0.84(95 \%$ confidence interval $(\mathrm{CI}): 0.69,1.00))$.

Maximum likelihood estimate of the constant parameter $k$ was 0.044 (95\% CI: $0.031,0.059)$.

Sensitivity and specificity were estimated at 77.5\% (95\% CI: 64.6, 90.4) and $85.9 \%(95 \%$ CI: risks of importation are identified in South America and western part of European countries.

(Figure 3), 18 countries (60\%) had already imported ZIKV before week 46.

202 transmission. Comparing predictive performance by AUC among models with all possible combinations of three explanatory variables, the model with CHIKV and DENV appeared to

204 yield the greatest value of AUC $(0.90$ (95\% CI: 0.60, 1.00)). Figures 1B and 1D show the 
205 distribution of the estimated risk and ROC curve, respectively. Using the best model, sensitivity

206 was as high as $96.4 \%(95 \%$ CI: 89.6, 100.0), while the specificity was estimated at $67.7 \%(95 \%$

207 CI: 60.5, 74.9). The second best model was the one with CHIKV only.

208

209

210

211

212

213

214

215

216

217

218

\section{Discussion}

220

221

222

223

224

225

226

Table 3 shows parameter estimates of the best fitted model. Compared with the absence of Chikungunya virus, the risk of local transmission in the presence of this virus was shown to be indicative of 22.9 times $(95 \%$ CI: 3.3, 238.3) higher. The presence of dengue was not statistically significant, but the adjusted odds ratio was estimated to be 7.7 (95\% CI: 1.0, 73.6). Table 4 shows correlations between two dichotomous variables as measured by Cohen's kappa. No particular correlation that could lead to multi-collinearity (e.g. kappa $>0.60$ ) was identified.

Figure 2B shows the country-specific global distribution of local transmission using the best model. The high risk of local transmission is seen mainly among countries in tropical and subtropical areas. Figures 3B show the top 30 countries with high risk of local transmission by week 92, using Brazil as the origin of spread. Among the total, 19 countries $(63.3 \%)$ were predicted to have already allowed local transmission before week 46 .

The present study estimated country-specific risk of importation and local transmission of ZIKV infection using a simple statistical model. As reported elsewhere, ZIKV infection was often internationally spread by mildly infected travellers (Tappe et al., 2013; Kutsuna et al., 2014; Korhonen et al., 2016). Potentially high importation risk in many temperate countries has motivated us to explore the risks of importation and local transmission fuelled by travellers. Our model is not as sophisticated as mapping precise risk of transmission using seasonal population dynamics of Aedes species and temperature/climatological data (Bogoch et al., 2016; Nah et al., 
227 2016), but the findings at country levels from our study are broadly consistent with what has

228 been briefly described at finer scales (Bogoch et al., 2016). Without using finer scale spatial data

229 (e.g. ecological data on Geographic Information Systems), our approach has crudely and clearly

230 distinguished the risk of local transmission from importation risk at country levels using a more

231 tractable approach. We have shown that the predicted risk of local transmission was frequently

232 seen in tropical and subtropical countries with DENV or CHIKV epidemic experience, while the 233 risk of importation was more scattered around the world. The diagnostic performance of risk

234 model for local transmission was well supported by AUC value of 0.90 .

Our study contributes to the risk assessment practice at each country level using

estimated risks of importation and local transmission expressed as probabilities. We have shown

237 that the risk of importation may be high in several countries given continued global travel of mildly infected travellers. However, considering that the public health concerns over ZIKV infection stems from the presence of microcephaly, it is more important to focus on the risk of local and widespread transmission that could involve pregnant women. Compared with the risk

241 of importation, the risk of local transmission was particularly highlighted in countries with

242 DENV and CHIKV epidemic experience. Considering that the Olympic Game in Brazil 2016

243 will elevate the risk of ZIKV to spread over wider spatial extent (Petersen et al., 2016), the

244 distinction between importation and local transmission will be even more important than it has

245 been recognized. Whereas our risk model of local transmission relied on the presence of

246 transmission by DENV or CHIKV, the use of such dataset should be deemed only as a proxy.

247 The sensitivity of our best model was $96.4 \%$, and thus, there is limited case in which the absence

248 of DENV or CHIKV lead to the local transmission of ZIKV. However, the specificity was

249 limited to $67.7 \%$, indicating that there would be a number of countries in which ZIKV 
250 transmission has yet to occur even in the presence of DENV and CHIKV. Our simple model

251 must have missed important additional predictors of local transmission in this regard, and such

252 predictors have to be sought in the future studies. Moreover, to further improve the prediction,

253 ecological data of vector behaviour at finer spatial scale must be incorporated with validation

254 using epidemic data.

A few limitations must be discussed. First, ascertainment bias cannot be ignored for

256

257

258

259

260

261

262

263

264

265

266

267

268

269

270

271

data in Table 1 must have missed several importation events. Presented risks of importation and

local transmission were thus probably underestimated on a whole. Second, the network data we used were static, and the airline transportation data represented only the number of flight routes (i.e. not the number of passengers). Nevertheless, assuming that the geographic patterns of travel have been maintained and only the travel volume has changed over short period of time, our simplistic approach is still justifiable to predict the global spread from 2015. Third, countryspecific modelling exercise suffers from heterogeneity within each single country. For instance, the United States, China and Australia have both high and low risk areas of transmission due to vast land with different climatological areas. Finally, we estimated the importation risk of ZIKV in a country as a risk of importing ZIKV from Brazil to the country. Therefore, our model cannot capture the risk of importing ZIKV from other endemic area such as South Pacific.

Despite clear need to improve predictions in the future, the present study successfully devised a simple global risk prediction of importation and local transmission. Countries with DENV and CHIKV epidemic experience are likely to be at particular high risk and such countries should be prepared for vector control measures such as avoiding daytime biting and the 
272 use of mosquito repellent. To further improve model predictions, it is essential to have laboratory

273 capacity built up in every single country at risk of local transmission.

\section{Conclusions}

275 Risks of importation and local transmission of Zika virus infection were estimated, analyzing

276 epidemiological, ecological and mobility data. Whereas the risk of importation was well

277 explained by the airline transportation network data, the risk of local transmission appeared to be

278 best captured by additionally accounting for the presence of an epidemic of Dengue and

279 Chikungunya viruses.

\section{References}

282

283

284

285

286

287

288

289

290

291

292

Adelman ZN, Tu Z. 2016. Control of Mosquito-Borne Infectious Diseases: Sex and Gene Drive.

Trends in Parasitology 32(3): 219-229.

Anonymous. 2016. Controlling Dengue Outbreaks. Scitable, Available at:

http://www.nature.com/scitable/topicpage/controlling-dengue-outbreaks-22403714 (last accessed on 15 March 2016)

Babaniyi OA, Mwaba P, Songolo P, Mazaba-Liwewe ML, MweeneNdumba I, Masaninga F. 2015. Seroprevalence of Zika virus infection specific IgG in Western and North-Western Provinces of Zambia. International Journal of Public Health and Epidemiology. 4(1): 110-114. Benelli G. 2016. Plant-mediated biosynthesis of nanoparticles as an emerging tool against mosquitoes of medical and veterinary importance: a review. Parasitology Research 115(1): 2334. 
293 Benelli G, Mehlhorn H. 2016. Declining malaria, rising of dengue and Zika virus: insights for

294 mosquito vector control. Parasitology Research, in press (doi: 10.1007/s00436-016-4971-z).

295 Bogoch II, Brady OJ, Kraemer MU, German M, Creatore MI, Kulkarni MA, Brownstein JS,

296 Mekaru SR, Hay SI, Groot E, Watts A, Khan K. 2016. Anticipating the international spread of

297 Zika virus from Brazil. The Lancet, 387(10016):335-6.

298 Brasil P, Pereira JP Jr, Raja Gabaglia C, Damasceno L, Wakimoto M, Ribeiro Nogueira RM,

299 Carvalho de Sequeira P, Machado Siqueira A, Abreu de Carvalho LM, Cotrim da Cunha D,

300 Calvet GA, Neves ES, Moreira ME, Rodrigues Baião AE, Nassar de Carvalho PR, Janzen C,

301 Valderramos SG, Cherry JD, Bispo de Filippis AM, Nielsen-Saines K. 2016. Zika Virus

302 Infection in Pregnant Women in Rio de Janeiro - Preliminary Report. New England Journal of

303 Medicine, in press (doi: 10.1056/NEJMoa1602412)

304 Brockmann D, Helbing D. 2013 .The hidden geometry of complex, network-driven contagion

305 phenomena. Science. 342(6164):1337-42.

306 Campos GS, Bandeira AC, Sardi SI. 2015. Zika Virus Outbreak, Bahia, Brazil. Emerging

307 Infectious Diseases. 21(10):1885-6. doi: 10.3201/eid2110.150847.

308 Centers for Disease Control and Prevention (CDC), USA. 2016a. Chikungunya virus,

309 Geographic Distribution. Available at: http://www.cdc.gov/chikungunya/geo/ (accessed on: 7

310 March 2016)

311 Centers for Disease Control and Prevention (CDC), USA. 2016b. Dengue homepage. Available

312 at: http://www.cdc.gov/dengue/epidemiology/ (accessed on: 7 March 2016)

313 Contentshare. 2016. Openflight database. Singapore: Contentshare. Available at:

314 http://openflights.org (accessed on: 28 January 2016) 
315 Dick GW. 1952. Zika virus. II. Pathogenicity and physical properties. Transactions of the Royal

316 Society of Tropical Medicine and Hygiene, 46: 521-534. doi: 10.1016/0035-9203(52)90043-6

317 European Centre for Disease Prevention and Control (ECDC). 2016. Countries and territories

318 with local Zika transmission. Available at:

319 http://ecdc.europa.eu/en/healthtopics/zika_virus_infection/zika-outbreak/Pages/Zika-countries-

320 with-transmission.aspx (accessed on 7 March 2016)

321 Gatherer D, Kohl A. 2016. Zika virus: a previously slow pandemic spreads rapidly through the

322 Americas. Journal of General Virology, 97(2):269-73.

323 Gengsheng Q, Hotilovac L. 2008. Comparison of non-parametric confidence intervals for the

324 area under the ROC curve of a continuous-scale diagnostic test. Statistical Methods in Medical

325 Research, 17: 207-221.

326 Global Flights Network. 2016. USA: General Electric Company and Seed Media Group.

327 Available at: http://www.visualizing.org/datasets/global-flights-network (accessed on: 28

328 January 2016)

329 Greiner M, Pfeiffer D, Smith RD. 2000. Principles and practical application of the receiver-

330 operating characteristic analysis for diagnostic tests. Preventive Veterinary Medicine, 45: 23-41.

331 Ioos S, Mallet HP, Leparc Goffart I, Gauthier V, Cardoso T, Herida M. 2014. Current Zika virus

332 epidemiology and recent epidemics. Médecine et maladies infectieuses. 44(7):302-307.

333 Korhonen EM, Huhtamo E, Smura T, Kallio-Kokko H, Raassina M, Vapalahti O. 2016. Zika

334 virus infection in a traveller returning from the Maldives, June 2015. Eurosruveillance 21(2);

335 doi: 10.2807/1560-7917.ES.2016.21.2.30107.

336 Kraemer MU, Sinka ME, Duda KA, Mylne A, Shearer FM, Brady OJ, Messina JP, Barker CM,

337 Moore CG, Carvalho RG, Coelho GE, Van Bortel W, Hendrickx G, Schaffner F, Wint GR, 
338 Elyazar IR, Teng HJ, Hay SI. 2015. The global compendium of Aedes aegypti and Ae.

339 albopictus occurrence. Scientific Data 2:150035.

340 Kutsuna S, Kato Y, Takasaki T, Moi M, Kotaki A, Uemura H, Matono T, Fujiya Y, Mawatari M,

341 Takeshita N, Hayakawa K, Kanagawa S, Ohmagari N. 2014. Two cases of Zika fever imported

342 from French Polynesia to Japan, December 2013 to January 2014. Eurosurveillance 19(4);

343 pii $=20683$.

344 Kwong JC, Druce JD, Leder K. 2013. Zika virus infection acquired during brief travel to

345 Indonesia. American Journal of Tropical Medicine and Hygiene. 89(3):516-7. doi:

346 10.4269/ajtmh.13-0029.

347 Mlakar J, Korva M, Tul N, Popović M, Poljšak-Prijatelj M, Mraz J, Kolenc M, Resman Rus K,

348 Vesnaver Vipotnik T, Fabjan Vodušek V, Vizjak A, Pižem J, Petrovec M, Avšič Županc T.

349 2016. Zika virus associated with microcephaly. New England Journal of Medicine, in press (doi:

350 10.1056/NEJMoa1600651).

351 Musso D, Cao-Lormeau VM, Gubler DJ. 2015. Zika virus: following the path of dengue and

352 chikungunya? Lancet. 386(9990):243-244.

353 Nah K, Otsuki S, Chowell G, Nishiura H. 2016. Predicting the international spread of Middle

354 East respiratory syndrome (MERS). BMC Infectious Diseases, in press.

355 Nishiura H, Kinoshita R, Mizumoto K, Yasuda Y, Nah K. 2016a. Transmission potential of Zika

356 virus infection in the South Pacific. International Journal of Infectious Diseases, in press (doi:

357 10.1016/j.ijid.2016.02.017)

358 Nishiura H, Mizumoto K, Rock KS, Yasuda Y, Kinoshita R, Miyamatsu Y. 2016b. A theoretical

359 estimate of the risk of microcephaly during pregnancy with Zika virus infection. Epidemics, in

360 press (doi: 10.1016/j.epidem.2016.03.001) 
361 Petersen E, Wilson ME, Touch S, McCloskey B, Mwaba P, Bates M, Dar O, Mattes F, Kidd M,

362 Ippolito G, Azhar EI, Zumla A. 2016. Rapid Spread of Zika Virus in The Americas -

363 Implications for Public Health Preparedness for Mass Gatherings at the 2016 Brazil Olympic

364 Games. International Journal of Infectious Diseases 44:11-15.

365 Staples JE, Dziuban EJ, Fischer M, Cragan JD, Rasmussen SA, Cannon MJ, Frey MT, Renquist

366 CM, Lanciotti RS, Muñoz JL, Powers AM, Honein MA, Moore CA. 2016. Interim Guidelines

367 for the Evaluation and Testing of Infants with Possible Congenital Zika Virus Infection - United

368 States, 2016. Morbidity and Mortality Weekly Report. 65(3):63-67.

369 Tappe D, Rissland J, Gabriel M, Emmerich P, Gunther S, Held G, Smola S, Schmidt-Chanasit J.

370 2014. First case of laboratory-confirmed Zika virus infection imported into Europe, November

371 2013. Eurosurveillance, 19(4); pii=20685.

372 The Walter Reed Biosystematics Unit, USA. 2016. Aedes africana. Available at:

373 http://www.wrbu.org/SpeciesPages_non-ANO/non-ANO_A-hab/AEafr_hab.html (accessed on: 7

374 March 2016)

375 World Health Organization, Western Pacific Region (WPRO). 2016. Pacific syndromic

376 surveillance report. Manila, WPRO. Available at: http://www.wpro.who.int/southpacific/en/

377 (accessed on 31 January 2016). 
380

381

382

383

384

385

386

387

388

389

390

391

392

393

394

395

396

397

398

399

400

\section{Figure legends}

\section{Figure 1. Predicted risks of ZIKV infection}

A - B) Distribution of estimated risks of importation and local transmission by country. The effective distance was used for panel A, while the presence of dengue and chikungunya viruses was additionally considered for panel B. C - D) Receiver operator characteristic curves of predicted risks of importation and local transmission. Panel $\mathrm{C}$ shows the evaluation results of the risk of importation that rested on the effective distance from Brazil, while D shows the risk of local transmission additionally accounted for dengue and chikungunya virus epidemic data.

Figure 2. Global distribution of risks of importation and local transmission with Zika virus

A. The importation risk of ZIKV by week 92 is colored by intensity $(0-20 \%, 20-40 \%, 40-60 \%$, $60-80 \%)$. The origin country, Brazil, is colored in grey. Other additional countries colored in grey were excluded, because they experienced importation of ZIKV infection prior to the event in Brazil (week 12, 2015). B. The local transmission risk of ZIKV infection by week 92 accounting for dengue and chikungunya epidemic data. The local transmission risk of ZIKV is colored by intensity $(0-15 \%, 15-30 \%, 30-45 \%, 45-60 \%)$. The origin country, Brazil, is colored in grey. Other additional countries colored in grey were excluded, because they experienced importation of ZIKV infection prior to the event in Brazil (week 12, 2015).

\section{Figure 3. Countries at high risk of ZIKV infection}

A-B) List of top 30 countries with the estimated highest risks. Panel A shows the risk of importation, while B shows the risk of local transmission. The risks shown on horizontal axes represent our estimates by the end of 2016 (week 92). Bars filled with grey represent countries 
401 that have already experienced importation of ZIKV infected case(s) by 31 January 2016 (week

402 46). 


\section{1}

Figure 1. Predicted risks of ZIKV infection

A - B) Distribution of estimated risks of importation and local transmission by country. The effective distance was used for panel $A$, while the presence of dengue and chikungunya viruses was additionally considered for panel B. C - D) Receiver operator characteristic curves of predicted risks of importation and local transmission. Panel $\mathrm{C}$ shows the evaluation results of the risk of importation that rested on the effective distance from Brazil, while $D$ shows the risk of local transmission additionally accounted for dengue and chikungunya virus epidemic data.
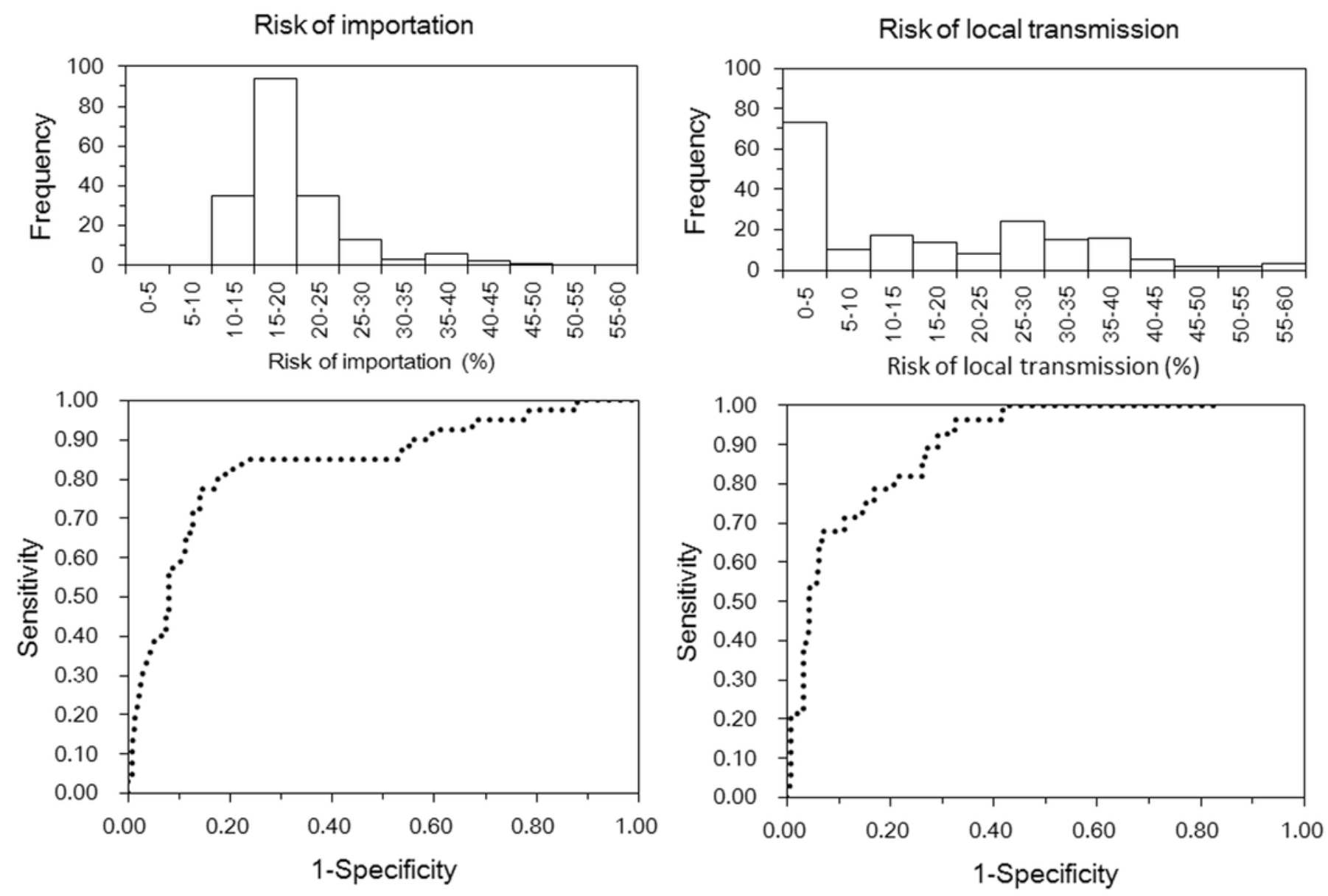


\section{2}

Figure 2. Global distribution of risks of importation and local transmission with Zika virus

A. The importation risk of ZIKV by week 92 is colored by intensity $(0-20 \%, 20-40 \%, 40-60 \%$, $60-80 \%)$. The origin country, Brazil, is colored in grey. Other additional countries colored in grey were excluded, because they experienced importation of ZIKV infection prior to the event in Brazil (week 12, 2015). B. The local transmission risk of ZIKV infection by week 92 accounting for dengue and chikungunya epidemic data. The local transmission risk of ZIKV is colored by intensity $(0-15 \%, 15-30 \%, 30-45 \%, 45-60 \%)$. The origin country, Brazil, is colored in grey. Other additional countries colored in grey were excluded, because they experienced importation of ZIKV infection prior to the event in Brazil (week 12, 2015). 


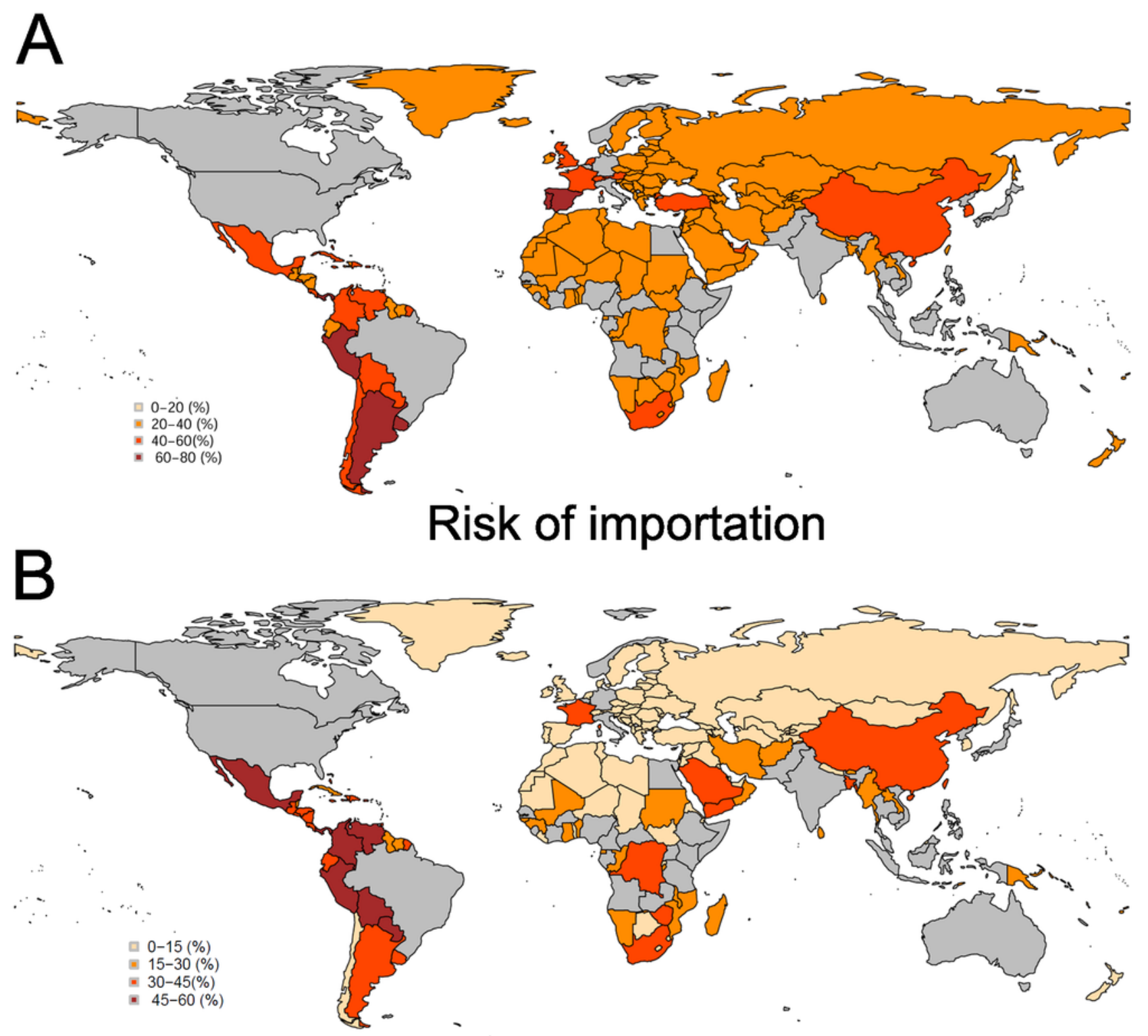

Risk of local transmission 
Figure 3. Countries at high risk of ZIKV infection

A-B) List of top 30 countries with the estimated highest risks. Panel A shows the risk of importation, while B shows the risk of local transmission. The risks shown on horizontal axes represent our estimates by the end of 2016 (week 92). Bars filled with grey represent countries that have already experienced importation of ZIKV infected case(s) by 31 January 2016 (week 46).
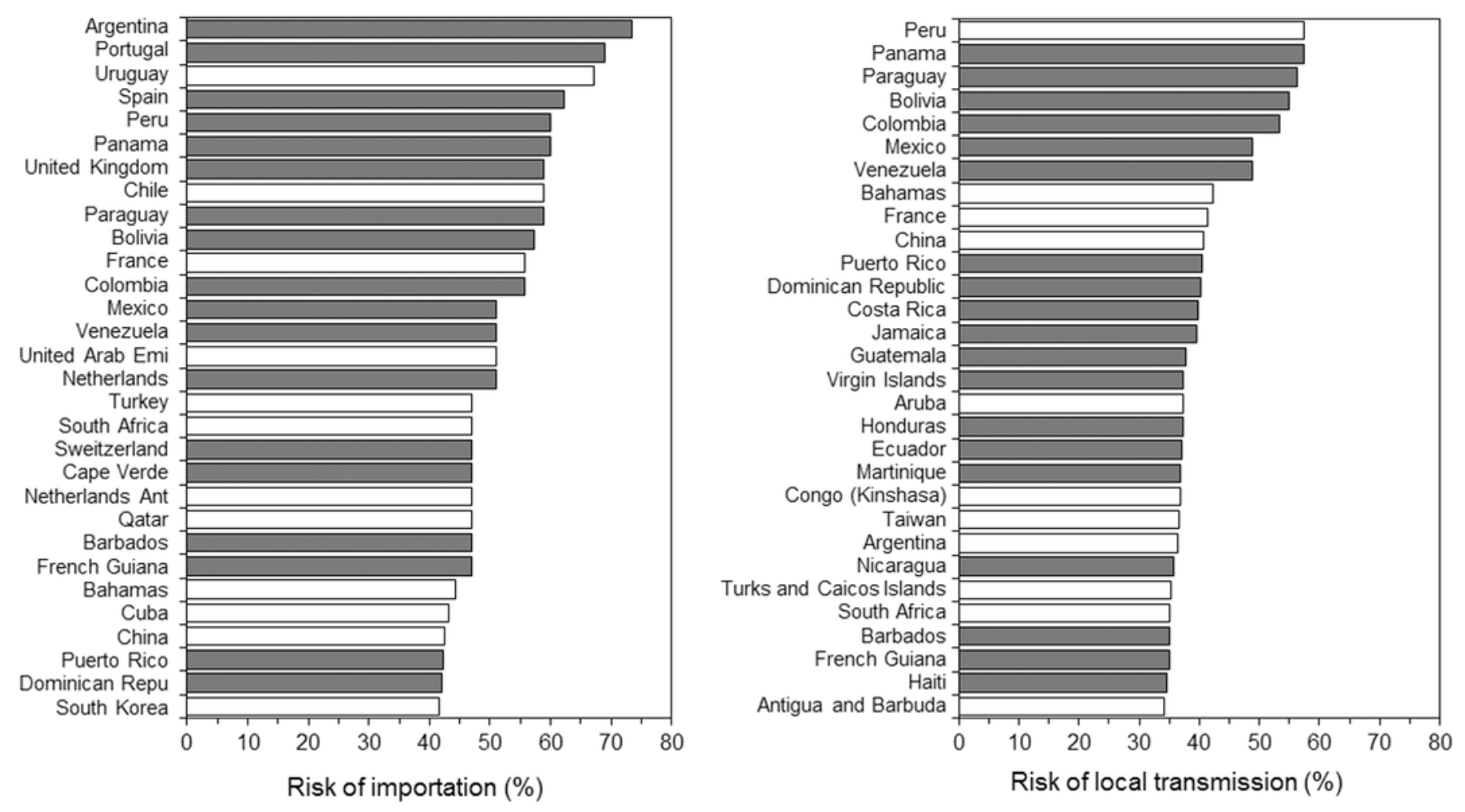


\section{Table $\mathbf{1}$ (on next page)}

Table 1. The list of countries that experienced importation of Zika virus infection

The earliest date at which an infected individual has likely to have entered the country is shown as the week counting from week 26, 1946. Extracting the dataset from World Health Organization source, the week number of the report has been originally written and we used it as the week of importation. When the exact week was unavailable, the mid-point of available time window was used as the week of importation. The first day of the year was set to be the first Sunday of January. One year was calculated to be exactly equal to 52 weeks in our analysis. Although three confirmed cases of ZIKV infection were reported in Chile on 2 February 2016, that data was ignored in the present study, because the latest time was set at fourth week of 2016. We also did not count a case report from Easter Island, a Chilean island in the southeastern Pacific Ocean, because the island is geographically distant from other parts of Chile and has only one airport (World Health Organization, 2016). In Australia, although one imported case is reported, there was no available sources of information to identify the date of confirmation. Instead, given that the corresponding article was received on 16 January 2013, we consider that the importation event took place in the previous year, 2012 and took the mid-point of the year as the week of importation (Kwong, Druce $\&$ Leder, 2013). In Zambia, a cross sectional study was conducted, but there was no available information to identify the date of importation or survey. Thus, the year of the acceptance of the article, 2015 was assumed as the year of importation (Babaniyi et al., 2015). 
1 Table 1. The list of countries that experienced importation of Zika virus infection

\begin{tabular}{|c|c|c|c|c|c|c|c|}
\hline Country & Year & Week & $\begin{array}{l}\text { Weeks } \\
\text { since } \\
\text { Uganda }\end{array}$ & Country & Year & Week & $\begin{array}{l}\text { Weeks } \\
\text { since } \\
\text { Uganda }\end{array}$ \\
\hline Countries up to Brazil & & & & Countries after Brazil & & & \\
\hline Uganda & 1947 & 26 & 0 & Vanuatu & 2015 & 17 & 3527 \\
\hline Tanzania & 1948 & 26 & 52 & Sweden & 2015 & 28 & 3538 \\
\hline Indonesia & 1951 & 26 & 208 & Fiji & 2015 & 33 & 3543 \\
\hline Malaysia & 1951 & 26 & 208 & Samoa & 2015 & 37 & 3547 \\
\hline India & 1952 & 26 & 260 & Colombia & 2015 & 42 & 3552 \\
\hline Philippines & 1953 & 26 & 312 & Suriname & 2015 & 45 & 3555 \\
\hline Egypt & 1953 & 26 & 312 & El Salvador & 2015 & 47 & 3557 \\
\hline Thailand & 1954 & 26 & 364 & Guatemala & 2015 & 47 & 3557 \\
\hline Vietnam & 1954 & 26 & 364 & Mexico & 2015 & 48 & 3558 \\
\hline Angola & 1960 & 22 & 672 & Venezuela & 2015 & 48 & 3558 \\
\hline Kenya & 1967 & 26 & 1040 & Netherlands & 2015 & 48 & 3558 \\
\hline Ethiopia & 1967 & 26 & 1040 & Panama & 2015 & 48 & 3558 \\
\hline Somalia & 1967 & 26 & 1040 & Paraguay & 2015 & 48 & 3558 \\
\hline Gabon & 1967 & 26 & 1040 & Honduras & 2015 & 51 & 3561 \\
\hline Nigeria & 1968 & 26 & 1092 & Cape Verde & 2015 & 51 & 3561 \\
\hline $\begin{array}{l}\text { Central African } \\
\text { Republic }\end{array}$ & 1968 & 26 & 1092 & Spain & 2015 & 52 & 3562 \\
\hline Senegal & 1968 & 26 & 1092 & Puerto Rico & 2016 & 1 & 3563 \\
\hline Sierra Leone & 1972 & 26 & 1300 & Martinique & 2016 & 2 & 3564 \\
\hline Pakistan & 1980 & 26 & 1716 & French Guiana & 2016 & 2 & 3564 \\
\hline Cote d'Ivoire & 1980 & 26 & 1716 & Finland & 2016 & 2 & 3564 \\
\hline Burkina Faso & 1981 & 26 & 1768 & United Kingdom & 2016 & 3 & 3565 \\
\hline Micronesia & 2007 & 26 & 3120 & Taiwan & 2016 & 3 & 3565 \\
\hline United States & 2008 & 26 & 3172 & Bolivia & 2016 & 3 & 3565 \\
\hline Cameroon & 2010 & 26 & 3276 & Ecuador & 2016 & 3 & 3565 \\
\hline Cambodia & 2010 & 34 & 3284 & Haiti & 2016 & 3 & 3565 \\
\hline Australia & 2012 & 26 & 3380 & Guadeloupe & 2016 & 3 & 3565 \\
\hline Canada & 2013 & 5 & 3411 & Barbados & 2016 & 3 & 3565 \\
\hline Germany & 2013 & 26 & 3432 & Guyana & 2016 & 3 & 3565 \\
\hline French Polynesia & 2013 & 46 & 3452 & Argentina & 2016 & 4 & 3566 \\
\hline
\end{tabular}




\begin{tabular}{llll|llll} 
Japan & 2013 & 50 & 3456 & Peru & 2016 & 4 & 3566 \\
Norway & 2013 & 50 & 3456 & Portugal & 2016 & 4 & 3566 \\
Italy & 2014 & 1 & 3459 & Austria & 2016 & 4 & 3566 \\
New Caledonia & 2014 & 1 & 3459 & Costa Rica & 2016 & 4 & 3566 \\
Cook Islands & 2014 & 9 & 3467 & Switzerland & 2016 & 4 & 3566 \\
Solomon Islands & 2014 & 12 & 3470 & Dominican Republic & 2016 & 4 & 3566 \\
Zambia & 2014 & 26 & 3484 & Jamaica & 2016 & 4 & 3566 \\
Belgium & 2014 & 38 & 3496 & Denmark & 2016 & 4 & 3566 \\
Brazil & 2015 & 12 & 3522 & Virgin Islands & 2016 & 4 & 3566 \\
& & & & Nicaragua & 2016 & 4 & 3566 \\
& & & & Tonga & 2016 & 4 & 3566 \\
\hline
\end{tabular}

3 The earliest date at which an infected individual has likely to have entered the country is shown

4 as the week counting from week 26, 1946. Extracting the dataset from World Health

5 Organization source, the week number of the report has been originally written and we used it as

6 the week of importation. When the exact week was unavailable, the mid-point of available time

7 window was used as the week of importation. The first day of the year was set to be the first

8 Sunday of January. One year was calculated to be exactly equal to 52 weeks in our analysis.

9 Although three confirmed cases of ZIKV infection were reported in Chile on 2 February 2016,

10 that data was ignored in the present study, because the latest time was set at fourth week of 2016.

11 We also did not count a case report from Easter Island, a Chilean island in the southeastern

12 Pacific Ocean, because the island is geographically distant from other parts of Chile and has only

13 one airport (World Health Organization, 2016). In Australia, although one imported case is

14 reported, there was no available sources of information to identify the date of confirmation.

15 Instead, given that the corresponding article was received on 16 January 2013, we consider that

16 the importation event took place in the previous year, 2012 and took the mid-point of the year as

17 the week of importation (Kwong, Druce \& Leder, 2013). In Zambia, a cross sectional study was

18 conducted, but there was no available information to identify the date of importation or survey.

19 Thus, the year of the acceptance of the article, 2015 was assumed as the year of importation

20 (Babaniyi et al., 2015). 


\section{Table 2 (on next page)}

Table 2. Predictive performance of risk models of importation and local transmission

IAUC, area under the curve. The confidence intervals were calculated using Mann-Whitney method (Gengsheng \& Hotilovac, 2008). \#Cl, confidence interval. 
1 Table 2. Predictive performance of risk models of importation and local transmission

\begin{tabular}{|c|c|c|c|c|c|}
\hline ID & $\begin{array}{l}\text { Predicted risk } \\
\text { (variables) }\end{array}$ & $\begin{array}{l}\mathrm{AUC}^{1}(95 \% \\
\left.\mathrm{CI}^{\#}\right)\end{array}$ & $\begin{array}{l}\text { Cut-off } \\
(\%)\end{array}$ & $\begin{array}{l}\text { Sensitivity } \\
\left(95 \% \mathrm{CI}^{\#}\right)\end{array}$ & $\begin{array}{l}\text { Specificity } \\
\left(95 \% \mathrm{CI}^{\#}\right)\end{array}$ \\
\hline NA & Importation & $\begin{array}{l}0.84 \\
(0.69,1.00)\end{array}$ & 20.6 & $\begin{array}{l}77.5 \\
(64.6,90.4)\end{array}$ & $\begin{array}{l}85.9 \\
(80.3,91.5)\end{array}$ \\
\hline 1 & $\begin{array}{l}\text { Local transmission } \\
\text { (Aedes) }\end{array}$ & $\begin{array}{l}0.80 \\
(0.55,1.00)\end{array}$ & 16.2 & $\begin{array}{l}64.3 \\
(46.5,82.0)\end{array}$ & $\begin{array}{l}85.1 \\
(79.6,90.6)\end{array}$ \\
\hline 2 & $\begin{array}{l}\text { Local transmission } \\
\text { (Chikungunya) }\end{array}$ & $\begin{array}{l}0.89 \\
(0.62,1.00)\end{array}$ & 18.6 & $\begin{array}{l}71.4 \\
(54.7,88.2)\end{array}$ & $\begin{array}{l}93.2 \\
(89.3,97.1)\end{array}$ \\
\hline 3 & $\begin{array}{l}\text { Local transmission } \\
\text { (Dengue) }\end{array}$ & $\begin{array}{l}0.84 \\
(0.54,1.00)\end{array}$ & 17.4 & $\begin{array}{l}67.9 \\
(50.6,85.2)\end{array}$ & $\begin{array}{l}91.9 \\
(87.7,96.1)\end{array}$ \\
\hline 4 & $\begin{array}{l}\text { Local transmission } \\
\text { (Aedes \& } \\
\text { Chikungunya) }\end{array}$ & $\begin{array}{l}0.89 \\
(0.61,1.00)\end{array}$ & 14.7 & $\begin{array}{l}85.7 \\
(72.8,98.7)\end{array}$ & $\begin{array}{l}80.1 \\
(74.0,86.3)\end{array}$ \\
\hline 5 & $\begin{array}{l}\text { Local transmission } \\
\text { (Aedes \& Dengue) }\end{array}$ & $\begin{array}{l}0.86 \\
(0.55,1.00)\end{array}$ & 13.7 & $\begin{array}{l}82.1 \\
(68.0,96.3)\end{array}$ & $\begin{array}{l}74.5 \\
(67.8,81.3)\end{array}$ \\
\hline 6 & $\begin{array}{l}\text { Local transmission } \\
\text { (Chikungunya \& } \\
\text { Dengue) }\end{array}$ & $\begin{array}{l}0.90 \\
(0.60,1.00)\end{array}$ & 9.71 & $\begin{array}{l}96.4 \\
(89.6,100.0)\end{array}$ & $\begin{array}{l}67.7 \\
(60.5,74.9)\end{array}$ \\
\hline 7 & $\begin{array}{l}\text { Local transmission } \\
\text { (Aedes \& } \\
\text { Chikungunya \& } \\
\text { Dengue) }\end{array}$ & $\begin{array}{l}0.76 \\
(0.38,1.00)\end{array}$ & 51.2 & $\begin{array}{l}89.3 \\
(77.8,100.0)\end{array}$ & $\begin{array}{l}70.8 \\
(63.8,77.8)\end{array}$ \\
\hline
\end{tabular}

2 AUC, area under the curve. The confidence intervals were calculated using Mann-Whitney

3 method (Gengsheng \& Hotilovac, 2008). ${ }^{\#} \mathrm{CI}$, confidence interval. 


\section{Table 3(on next page)}

Table 3. Estimated parameters for describing the local transmission risk of Zika virus infection

NA, not applicable. Results from the best model that included Chikungunya and Dengue as explanatory variables are shown. Dependent nominal variable $=$ local transmission. $\mathrm{Cl}$ : confidence interval. 
1 Table 3. Estimated parameters for describing the local transmission risk of Zika virus infection

\begin{tabular}{ccc}
\hline & Estimated values (95\% CI) & Adjusted odds ratio (95\% CI) \\
\hline Intercept & $2.06(-4.23,-0.51)$ & NA \\
Chikungunya virus & $3.13(1.19,5.47)$ & $22.90(3.30,238.34)$ \\
Dengue virus & $2.04(-0.02,4.30)$ & $7.68(0.98,73.64)$ \\
\hline
\end{tabular}

2

3 NA, not applicable. Results from the best model that included Chikungunya and Dengue as

4 explanatory variables are shown. Dependent nominal variable $=$ local transmission. $\mathrm{CI}$ :

5 confidence interval.

6

7

8 


\section{Table 4(on next page)}

Table 4. Agreement statistic, kappa between two dichotomous variables

\# Cl: confidence intervals. 
1 Table 4. Agreement statistic, kappa between two dichotomous variables

2

\begin{tabular}{lc}
\hline Combination of variables & $\begin{array}{l}\text { Cohen's kappa, an agreement } \\
\text { statistic }\left(95 \% \mathrm{CI}^{\#}\right)\end{array}$ \\
\hline Aedes and Chikungunya & $0.442(0.327,0.556)$ \\
Aedes and Dengue & $0.366(0.235,0.496)$ \\
Chikungunya and Dengue & $0.523(0.405,0.642)$ \\
\hline
\end{tabular}

3

4 \# CI: confidence intervals.

5 